\title{
MODOS GUIADOS EM SLABS METAMATERIAIS
}

\section{GUIDED MODES IN METAMATERIAL SLABS}

\author{
Leonardo André Ambrosio ${ }^{1}$ Hugo E. Hernández-Figueroa ${ }^{1} \quad$ Héctor Torres-Silva $^{2}$
}

Recibido 20 de junio de 2006, aceptado 23 de octubre de 2006

Received: June 20, 2006 Accepted: October 23, 2006

\begin{abstract}
RESUMO
Este trabalho apresenta um estudo de revisão de modos propagantes em um guia-de-onda slab constituído de materiais com índices de refração negativo, os chamados metamateriais, Mostra-se que os modos guiados em um slab metamaterial possuem algumas propriedades particulares, tais como a propagação de ondas lentas simétricas ou anti-simétricas, a ausência de modos fundamentais para ondas rápidas e a possibilidade de propagação de ondas guiadas em um meio menos denso. A análise é baseada em expansões de campo no guia e nos espaços superior e inferior ao mesmo.
\end{abstract}

Palavras chaves: Metamateriais, modos guiados, índice de refração negativo, equações de Maxwell.

\section{ABSTRACT}

This paper presents a review of the propagation modes in a slab waveguide consisting of negative refraction index materials, known as metamaterials. Some particular properties of guided modes in a metamaterial slab, such as slow simmetric or antisimmetric slow wave propagation, the absence of fundamental modes for fast waves and the possibility of guided waves in a less dense medium. The analysis is based on field expansions in the guide and the upper and lower spaces of it.

Keywords: Metamaterial, guided modes, negative index of refraction, Maxwell's equations.

\section{INTRODUÇÃO}

Demonstrações experimentais recentes de novos materiais compostos com um índice de refração negativo abrem um novo caminho no projeto de novos tipos de dispositivos onde ondas eletromagnéticas se propagam de um modo não-convencional. A história de tais materiais inicia-se com o artigo de Veselago [1], o qual estuda a propagação de ondas em um hipotético material com, simultaneamente, permissividade dielétrica $\varepsilon$ e permeabilidade magnética $\mu$ negativos. Tais meios são usualmente chamados de metamateriais, ou "left-handed materials" (LHM), hajam visto os campos elétrico e magnético formarem uma tríade à esquerda com o vetor de onda, ao contrário dos convencionais "right-handed materials" (RHM). Em 1968, Veselago predisse algumas propriedades dos metamateriais, incluindo refração negativa. Entretanto, as estruturas com $\varepsilon$ e $\mu$ negativos não eram conhecidas até recentemente, quando as primeiras demonstrações experimentais de tais materiais foram feitas.
Uma das primeiras aplicações dos metamateriais foi sugerida por Pendry [2], o qual demonstrou que um slab composto por um material com índice de refração negativo e sem perdas poderia fornecer uma imagem perfeita de uma fonte pontual. Essa significativa melhora na resolução da imagem pode ser explicada pela excitação de ondas de superfície em ambas as interfaces do slab. Portanto, faz-se importante estudar as propriedades de ondas de superfície nas interfaces entre a refração negativa e materiais convencionais. No caso de plasmas infinitos com quiralidade estuda-se pela primeira vez casos de velocidade de fase negativa [3].

Neste artigo, estuda-se a estrutura e propriedades básicas de ondas guiadas por metamateriais inserido em um meio convencional com $\varepsilon$ e $\mu$ positivos, e vice-versa. De maneira a enfatizar as propriedades não usuais de tais ondas, é feita uma comparação com guias-deondas planares dielétricos convencionais. Mostra-se que os modos guiados em guias-de-onda metamateriais

\footnotetext{
1 Faculdade de Engenharia Elétrica e de Computação - FEEC - UNICAMP - Cidade Universitária Zeferino Vaz. Av. Albert Einstein, 400 CEP: 13083-970 - Barão Geraldo - Campinas - Brasil.

2 Instituto de Alta Investigación. Universidad de Tarapacá. Arica, Chile. Casilla 6-D.
} 
diferem drasticamente dos modos guiados convencionais, demonstrando que para números de onda transversais reais, os modos guiados não apresentam modos fundamentais, ao contrário de slabs convencionais em que existe sempre um modo fundamental par, e que para números de onda transversais puramente imaginários, existe a possibilidade de propagação, com ou sem cortes.

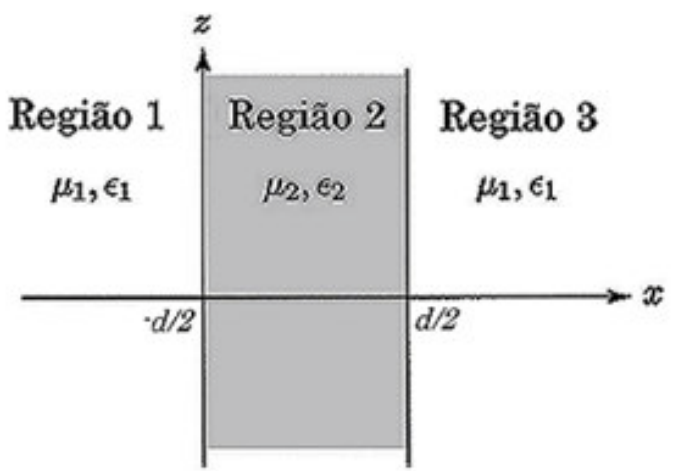

Figura 1. Slab simétrico. Meios 1 (Regiões 1 e 3) e 2 (Região 2) podem possuir parâmetros de permissividade e permeabilidade tanto positivos quanto negativos. Adaptado da figura 1 de [4].

\section{ANÁLISE TEÓRICA}

\section{Soluções de Modos Guiados}

Considere uma seção longitudinal de um slab simétrico sem perdas, onde tanto o meio 1 quanto o meio 2 podem ser constituídos por parâmetros $\mu$ e $\varepsilon$ negativos, conforme mostra a figura 1. Por simplicidade, é considerada uma não-dependência na coordenada $y$, o que nos leva a campos elétricos para modos TE (resultados para ondas TM podem ser obtidos usando dualidade) nas três regiões ilustradas da forma [4-7].

$$
\begin{aligned}
& E_{y}^{1}(x, z)=E_{1} e^{-\alpha_{1} x} e^{-j k_{z} z} \\
& E_{y}^{2}(x, z)=\left[A e^{-j k_{2 x} x}+B e^{j k_{2 x} x}\right] e^{-j k_{z} z} \\
& E_{y}^{3}(x, z)=E_{3} e^{\alpha_{1} x} e^{-j k_{z} z}
\end{aligned}
$$

onde $k_{z}$ é o vetor de onda real na direção $\hat{z}, \alpha_{1}$ a constante de atenuação nas regiões 1 e 3 e $k_{2 x}$ o número de onda transversal na região 2 , o qual pode ser tanto real quanto imaginário, e para $A=B$ temos os modos pares, enquanto que $A=-B$ determina modos ímpares.

Das equações de Maxwell, encontramos os campos magnéticos associados:

$$
\begin{aligned}
\vec{H}^{1}(x, z) & =\left(\frac{E_{1}}{\omega \mu_{1}}\right)\left[-\hat{x} k_{z}-\hat{z} j \alpha_{1}\right] e^{-\alpha_{1} x} e^{-j k_{z} z} \\
\vec{H}^{2}(x, z) & =\left(\frac{A+B}{\omega \mu_{2}}\right)\left[-\hat{x} k_{z} \cos \theta-\hat{z} j k_{2 x} \sin \theta\right] e^{-j k_{z} z} \\
& +\left(\frac{A-B}{\omega \mu_{2}}\right)\left[-\hat{x} j k_{z} \sin \theta-\hat{z} k_{2 x} \cos \theta\right] e^{-j k_{z} z} \\
\vec{H}^{3}(x, z) & =\left(\frac{E_{3}}{\omega \mu_{1}}\right)\left[-\hat{x} k_{z}+\hat{z} j \alpha_{1}\right] e^{\alpha_{1} x} e^{-j k_{z} z}
\end{aligned}
$$

Para encontrarmos as condições de guiamento, lembramos que os campos tangenciais devem ser contínuos nas interfaces $x=-d / 2$ e $d / 2$, obtendo

$$
\begin{aligned}
& E_{1} e^{-\alpha_{1} d / 2}=\left[(A+B) \cos \theta_{1}+j(A-B) \sin \theta_{1}\right] \\
& p_{21} E_{1} e^{-\alpha_{1} d / 2}=\left[(A-B) \cos \theta_{1}+j(A+B) \sin \theta_{1}\right] \\
& E_{3} e^{-\alpha_{1} d / 2}=\left[(A+B) \cos \theta_{1}-j(A-B) \sin \theta_{1}\right] \\
& -p_{21} E_{3} e^{-\alpha_{1} d / 2}=\left[(A-B) \cos \theta_{1}-j(A+B) \sin \theta_{1}\right] \\
& \text { onde } p_{21}=\frac{\mu_{2} j \alpha_{1}}{\mu_{1} k_{2 x}} \text { e } \theta_{1}=\frac{k_{2 x} d}{2} .
\end{aligned}
$$

Considerando, por simetria, somente a interface em $x=\mathrm{d} / 2$ e resolvendo (7) e (8) para ambos os modos TE pares e ímpares encontramos, respectivamente, as condições de guiamento para o caso em que o número de onda transversal $k_{2 x}$ é real:

$$
\begin{aligned}
& \left(\frac{\alpha_{1} d}{2}\right)=\frac{\mu_{1}}{\mu_{2}}\left(\frac{k_{2 x} d}{2}\right) \tan \left(\frac{k_{2 x} d}{2}\right), \quad \operatorname{modos} \text { TE pares (11) } \\
& \left(\frac{\alpha_{1} d}{2}\right)=-\frac{\mu_{1}}{\mu_{2}}\left(\frac{k_{2 x} d}{2}\right) \cot \left(\frac{k_{2 x} d}{2}\right), \text { modos TE ímpares (12) }
\end{aligned}
$$

Por dualidade, encontramos para os modos TM

$$
\begin{aligned}
& \left(\frac{\alpha_{1} d}{2}\right)=\frac{\varepsilon_{1}}{\varepsilon_{2}}\left(\frac{k_{2 x} d}{2}\right) \tan \left(\frac{k_{2 x} d}{2}\right), \quad \operatorname{modos} \text { TM pares } \\
& \left(\frac{\alpha_{1} d}{2}\right)=-\frac{\varepsilon_{1}}{\varepsilon_{2}}\left(\frac{k_{2 x} d}{2}\right) \cot \left(\frac{k_{2 x} d}{2}\right), \text { modos TM ímpares }
\end{aligned}
$$

As relações de dispersão para ambos os modos TE e TM nos meios 1 e 2 valem, respectivamente, 


$$
\begin{aligned}
& \left(\frac{k_{z} d}{2}\right)^{2}-\left(\frac{\alpha_{1} d}{2}\right)^{2}=\left(\frac{k_{1} d}{2}\right)^{2} \\
& \left(\frac{k_{z} d}{2}\right)^{2}+\left(\frac{k_{2 x} d}{2}\right)^{2}=\left(\frac{k_{2} d}{2}\right)^{2}
\end{aligned}
$$

o que nos leva a um conjunto de círculos definidos por

$$
\left(\frac{k_{2 x} d}{2}\right)^{2}+\left(\frac{\alpha_{1} d}{2}\right)^{2}=\left(k_{2}^{2}-k_{1}^{2}\right)\left(\frac{d}{2}\right)^{2}
$$

$\operatorname{com} k_{1,2}^{2}=\omega^{2} \mu_{1,2} \varepsilon_{1,2}$

Tais modos em que o vetor de onda transversal é real são chamados de modos rápidos, haja visto suas velocidades de fase $\omega / k_{\mathrm{z}}$ serem maiores do que a velocidade de fase em um meio homogêneo com os mesmos parâmetros $\varepsilon_{2}$ e $\mu_{2}$.

Embora modos rápidos sempre apresentem cortes para um slab LHM, a energia pode ainda propagar-se em qualquer freqüência devido à existência de outros tipos de modos, os modos lentos, os quais podem ou não ter cortes. Se o número de onda transversal for imaginário ( $k_{2 x}=-j \alpha_{2}, \operatorname{com} \alpha_{2}$ real positivo), o que acontece quando a constante de propagação $k_{\mathrm{z}}$ excede $k_{2}$, a condição de guiamento pode ser reescrita como

$$
\left(\frac{\alpha_{1} d}{2}\right)=-\frac{\mu_{1}}{\mu_{2}}\left(\frac{\alpha_{2} d}{2}\right) \tanh \left(\frac{\alpha_{2} d}{2}\right), \quad \text { modos TE pares }
$$

$$
\left(\frac{\alpha_{1} d}{2}\right)=-\frac{\mu_{1}}{\mu_{2}}\left(\frac{\alpha_{2} d}{2}\right) \operatorname{coth}\left(\frac{\alpha_{2} d}{2}\right), \quad \text { modos TE ímpares }
$$

para modos TE e, se substituirmos $\mu_{1,2}$ por $\varepsilon_{1,2}$ nas equações acima, temos as novas condições de guiamento para modos TM. As relações de dispersão nos dois meios se concatenam, novamente para ambos os modos TE e TM em

$$
\left(\frac{\alpha_{1} d}{2}\right)^{2}-\left(\frac{\alpha_{2} d}{2}\right)^{2}=\left(k_{2}^{2}-k_{1}^{2}\right)\left(\frac{d}{2}\right)^{2}
$$

Soluções das equações transcendentais podem ser obtidas graficamente. Na figura 2 temos a solução TE para um slab convencional, com ambos os meios apresentando índice de refração positivo, onde as linhas sólidas representam o lado direito das equações (11) e (12), enquanto que as linhas tracejadas indicam a equação (15) para diferentes guias-de-onda, mas com a mesma razão $\mu_{1} / \mu_{2}$. Fica claro não haver modos lentos, sendo que a condição para modos guiados deve satisfazer, de (15), $k_{2}{ }^{2}>k_{1}^{2}$, isto é, o meio 2 deve ser mais denso para que tenhamos um ângulo crítico de reflexão total. Neste caso, existe sempre um modo guiado fundamental, cujo perfil não contém zeros.

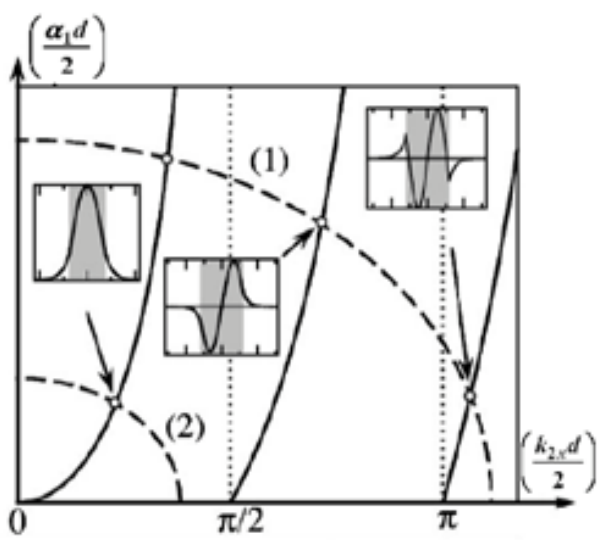

Figura 2. Modos guiados de um guia-de-onda convencional, com parâmetros de permissividade e permeabilidade positivos. Intersecções entre curvas tracejadas e sólidas indicam a existência de modos guiados. Os quadros interiores mostram os perfis transversais destes modos. Adaptado da figura 1 de [6].

Embora para um meio com índice de refração positivo o modo mais baixo não possua corte, há uma freqüência de corte para o menor modo em um slab LHM, como visto na figura 3 , representando em linhas sólidas o lado direito das equações (11-14) para $\mu_{1} / \mu_{2}<0$ no eixo $\left(k_{2 x} d / 2\right)$ e (18-19) no eixo $\left(\alpha_{2} d / 2\right)$, e as linhas tracejadas representam (20) para diferentes guiasde-ondas, mas com a razão $\mu_{1} / \mu_{2}<0$ fixa. Os cortes são determinados agora pela distância mínima entre a origem e as curvas tangente ou cotangente. Assim, a condição de corte é diferente daquela para um slab regular, o qual é determinado pela condição $\alpha_{1} d / 2=0$ (para modos propagantes).

As propriedades de guias-de-onda LHM são bem diferentes daquelas encontradas para slabs convencionais. Primeiro, porque tais guias-de-ondas podem suportar modos lentos, os quais podem ser tanto simétricos (pares) quanto anti-simétricos (ímpares), representando estas soluções estados de contorno em fase ou fora de fase de modos de superfície, localizados nas duas interfaces 
entre os meios LHM e RHM. No caso convencional de ambos $\mu$ e $\varepsilon$ positivos, as ondas de superfície não existem, aparecendo, contudo, quando a permeabilidade magnética muda de sinal (para polarização TE). Assim, modos guiados podem ser suportados por guias-de-ondas LHM de alto índice o de baixo índice.

Em segundo lugar, a hierarquia convencional de modos rápidos é rompida. Especificamente, (i) o modo fundamental de forma alguma existe, (ii) o modo de primeira ordem existe somente dentro de uma faixa de valores de $\left(k_{2} d / 2\right)$ e desaparece quando um valor crítico de $\left(k_{2 x} d / 2\right)$ é excedido e (iii) dois modos com o mesmo número de nós podem coexistir dentro do mesmo guia. Algumas destas características podem ser vistas na figura 3 .

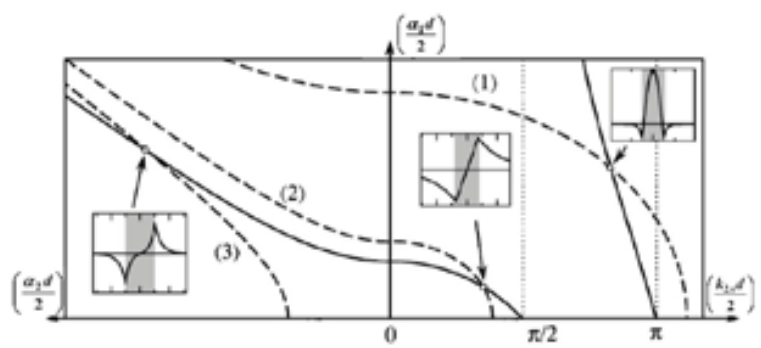

Figura 3. Determinação gráfica de $\left(k_{2 x} d / 2, \alpha_{2} d / 2\right)$ para slab metamaterial TE. Círculos indicam soluções para diferentes freqüências de operação. Note a configuração de onda de superfície no perfil transversal do modo lento. Adaptado da figura 1 de [6].

Uma outra visualização gráfica possível é unirmos (18) ou (19) com a equação (20) e esboçarmos $\left(k_{2}^{2}-k_{1}^{2}\right) d^{2}$ vs $\alpha_{2} d$ para os modos lentos simétrico e anti-simétrico, como mostrado nas figuras 4 e 5 , respectivamente.

Os eixos verticais nas figuras 4 e 5 correspondem aos valores de $\left(k_{2}^{2}-k_{1}^{2}\right) d^{2}$ ou $\omega^{2} \mu_{2} \varepsilon_{2}\left(1-\mu_{1} \varepsilon_{1} / \mu_{2} \varepsilon_{2}\right) d^{2}$, e os eixos horizontais correspondem aos valores de $\alpha_{2} d$. As regiões sombreadas correspondem a casos onde $k_{2}^{2}>k_{1}^{2}$ e as regiões não sombreadas, aos casos onde $k_{2}^{2}<k_{1}^{2}$. Note que, em ambos os casos, $k_{1}^{2}$ ou $k_{2}^{2}$ pode ser negativo, indicando um meio plasmático.

Para um conjunto específico de $\left(\mu_{1,2}, \varepsilon_{1,2}\right)$ e uma freqüência angular de operação $\omega$, podemos traçar uma linha horizontal nas figuras 4 e 5 onde as intersecções com as curvas de razão $-\mu_{1} / \mu_{2}$ nos fornece os valores de $\alpha_{2} d$, os quais caracterizam um modo propagante com número de onda transversal imaginário. Conforme a posição vertical desta linha horizontal varia com $\omega$, uma maior distância da linha a partir do 0 significa uma maior freqüência angular de operação. As freqüências de corte podem assim ser identificadas para meios com diferentes parâmetros constitutivos, e pode-se ver que os modos simétricos, quando presentes, não possuem corte, enquanto que modos anti-simétricos, dependendo dos parâmetros constitutivos, podem conter cortes.

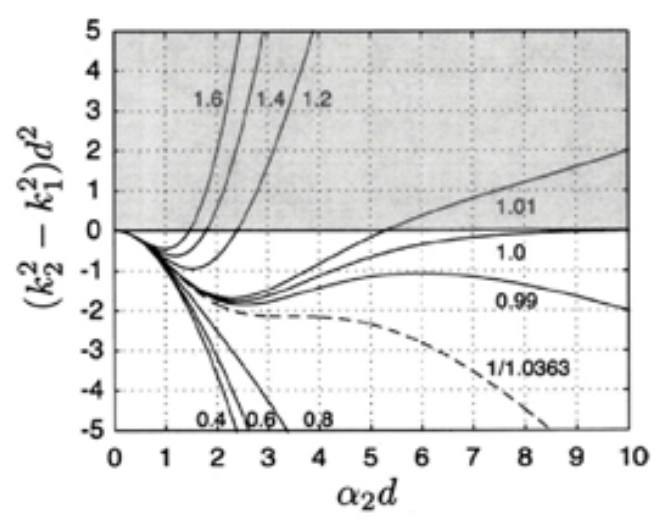

Figura 4. Determinação gráfica de $\alpha_{2} d$ para diferentes valores de $-\mu_{1} / \mu_{2}$ (indicados próximo à cada curva), modos TE lento simétrico. Adaptado da figura 4 de [4].

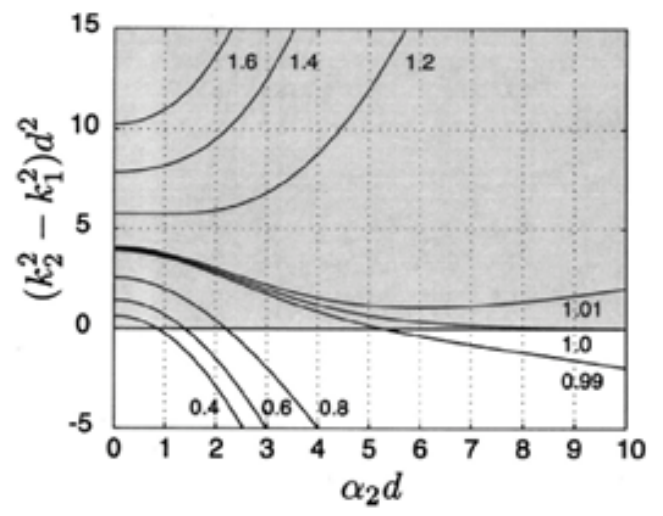

Figura 5. Determinação gráfica de $\alpha_{2} d$ para diferentes valores de $-\mu_{1} / \mu_{2}$ (indicados próximo à cada curva), modos TE lento anti-simétrico. Adaptado da figura 5 de [4].

A região não-sombreadas dos gráficos $\left[\left(k_{2}{ }^{2}-k_{1}{ }^{2}\right) d^{2}<0\right]$ corresponde a um slab opticamente menos denso, $\operatorname{com} k_{2}$ real, ou a um meio plasmático, $\operatorname{com} k_{2}$ sendo imaginário. Em ambos os casos, a expressão $\left(k_{2}^{2}-k_{1}^{2}\right) d^{2}$ é sempre negativa. A existência de modos anti-simétrico e simétrico para tais meios de plasma é bem conhecida [2], e o caso especial de um slab de plasma resulta nos mesmos modos cosh e sinh com de um slab com $k_{2}$ real negativo onde $\left|k_{2}\right|<\left|k_{1}\right|$. Portanto, as regiões inferiores dos gráficos $\left[\left(k_{2}{ }^{2}-k_{1}^{2}\right) d^{2}<0\right]$ representam o caso dual 
das soluções apresentadas em [5] (TE neste caso versus TM em [5]). Além disso, quando modos simétrico ou anti-simétrico existem dentro do guia, pode-se perceber que múltiplas intersecções são possíveis para certos parâmetros constitutivos para determinadas bandas de freqüência. A linha tracejada na figura 4, correspondendo $\mathrm{a}-\mu_{1} / \mu_{2}=1 / 1.0363$, representa o ponto onde a transição entre intersecção simples e intersecção múltipla ocorre. Tais resultados, aqui obtidos para modos TE, também são válidos para modos TM, além de serem os mesmos resultados que teríamos para o caso em que o meio 2 da figura 1 representa um meio convencional e o meio 1 o meio metamaterial com parâmetros constitutivos negativos.

Assim, para uma freqüência angular específica $\omega$ maior do que o corte do modo regular mais baixo mostrado nas figuras 2 e 3 , modos regulares rápidos coexistirão com modos lentos, de forma que devemos levá-los em consideração quando de um problema de guiamento.

\section{DISPERSÃO DE FREQÜÊNCIA}

A dispersão de freqüência de ondas guiadas em guiasde-ondas metamateriais deve ser estudada levando-se em conta a dispersão de ambos $\varepsilon_{2}$ e $\mu_{2}$-no caso em que o meio 2 na figura 1 é metamaterial-, haja visto esta dispersão ser uma propriedade essencial de tais materiais. Para tanto, consideram-se as seguintes dependências de freqüência das características efetivas dos meios metamateriais,

$$
\varepsilon_{2}(\omega)=1-\frac{\omega_{p}^{2}}{\omega^{2}}
$$

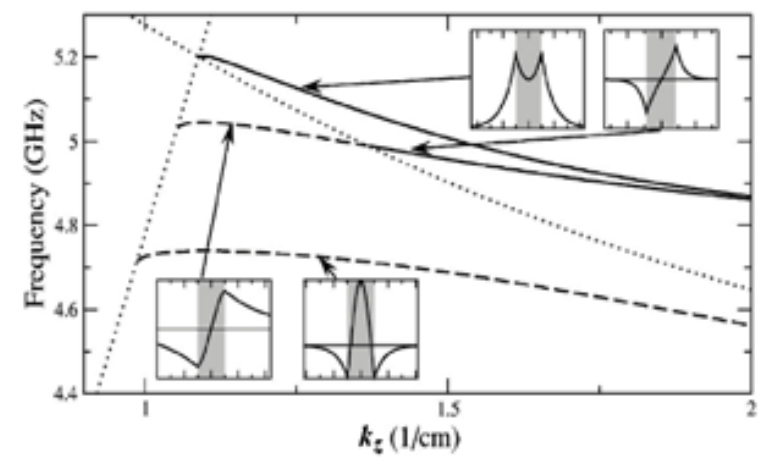

Figura 6. Curvas de dispersão de freqüência para os três modos guiados de menores ordens de um slab metamaterial $(d=2 \mathrm{~cm})$. As figuras interiores mostram os perfis transversais dos modos. Adaptado da figura 2 de [6].

$$
\mu_{2}(\omega)=1-\frac{F \omega^{2}}{\omega^{2}-\omega_{0}^{2}}
$$

onde os parâmetros $\omega_{1} / 2 \pi=10 \mathrm{GHz}, \omega_{0} / 2 \pi=4$ $\mathrm{GHz}$ e $F=0.56$. Tal escolha é motivada por resultados experimentais. A região em que tanto a permissividade quanto a permeabilidade são negativos é de $4 \mathrm{GHz}$ a 6 $\mathrm{GHz}$. Curvas de dispersão para os três primeiros modos guiados em um slab com espessura $d=2 \mathrm{~cm}$ são mostradas na figura 6 , onde as curvas tracejadas correspondem a modos rápidos e curvas sólidas a modos lentos. Notase que o modo lento fundamental existe apenas para freqüências mais altas, enquanto que modo rápido de segunda ordem aparece para freqüências mais baixas. Ambos os modos podem ter tanto dispersão de velocidade de grupo positiva quanto negativa em regiões de diferentes parâmetros. As propriedades do modo anti-simétrico de primeira ordem são diferentes. O tipo de modo muda, em determinado momento, de rápido para lento conforme $\mathrm{o}$ número de onda $k_{z}$ aumenta. Tal transição ocorre quando a condição $k_{2 x}=0$ é satisfeita, o que representa um contorno separando os dois tipos de modo, como mostrado na Fig. 6 por uma linha pontilhada. Os modos rápidos de ordem superior existem em freqüências próximas à freqüência de ressonância em $\omega=4 \mathrm{GHz}$.

\section{O VETOR DE POYTING E FLUXO DE ENERGIA}

Em metamateriais, as ondas eletromagnéticas são de retorno, já que o fluxo de energia e o vetor de onda são antiparalelos, enquanto que estes vetores são paralelos em materiais homogêneos convencionais (RHM). O Fluxo de energia é caracterizado pelo vetor de Poyting

$$
\vec{S}=\frac{1}{2} \operatorname{Re}\left[\vec{E} \times \vec{H}^{*}\right]
$$

Através de sua média em um período $T=2 \pi / \omega$. Um modo guiado tem, por definição, um perfil transversal estacionário, e o fluxo médio de energia é direcionado somente através do guia. Assim, a partir das equações de Maxwell e das equações (1-6), achamos as componentes $z$ do fluxo de energia para modos TE como sendo dadas por

$$
S_{z}^{1}=\frac{\left|E_{1}\right|^{2} k_{z}}{2 \omega \mu_{1}} e^{-2 \alpha_{1} x}
$$


$S_{z}^{2}= \begin{cases}\frac{2 A^{2} k_{z} \cos ^{2}\left(k_{2 x} x\right)}{\omega \mu_{2}} & , \text { modos pares } \\ \frac{2 A^{2} k_{z} \sin ^{2}\left(k_{2 x} x\right)}{\omega \mu_{2}} & , \text { modos ímpares }\end{cases}$

$S_{z}^{3}=\frac{\left|E_{3}\right|^{2} k_{z}}{2 \omega \mu_{1}} e^{2 \alpha_{1} x}$

para o caso em que $k_{2 x}$ é real e

$S_{z}^{1}=\frac{\left|E_{1}\right|^{2} k_{z}}{2 \omega \mu_{1}} e^{-2 \alpha_{1} x}$

$S_{z}^{2}= \begin{cases}\frac{2 A^{2} k_{z} \cosh ^{2}\left(\alpha_{2} x\right)}{\omega \mu_{2}} & , \text { modos pares } \\ \frac{2 A^{2} k_{z} \sinh ^{2}\left(\alpha_{2} x\right)}{\omega \mu_{2}} & , \text { modos ímpares }\end{cases}$

$S_{z}^{3}=\frac{\left|E_{3}\right|^{2} k_{z}}{2 \omega \mu_{1}} e^{2 \alpha_{1} x}$

O fluxo de potência total através dos meios 1 e 2 pode ser obtido de

$$
\begin{aligned}
& P_{1}=2 \int_{d / 2}^{\infty} \overline{S_{z}^{1}} d x \\
& P_{2}=\int_{-d / 2}^{d / 2} \overline{S_{z}^{2}} d x
\end{aligned}
$$

Estes resultados obtidos valem para guias cujo meio 2 é metamaterial e o meio 1 é convencional, ou vice-versa. Pode-se notar que a distribuição do fluxo de energia para ondas guiadas através de um slab para estes dois casos é de fato não-usual. De fato, o fluxo de energia dentro do slab é oposto àquele fora do mesmo. Isto ocorre porque a componente do vetor de onda normalizado através do guia $\left(k_{z}\right)$ é fixa em um modo guiado de acordo com as eqs. (1-3). Uma informação importante sobre os modos guiados pode ser extraída do fluxo de energia normalizado,

$$
p=\frac{P_{1}+P_{2}}{\left|P_{1}\right|+\left|P_{2}\right|}
$$

que possui os limites $|p|<1, p \rightarrow 1$ quando o modo é fracamente localizado, $\left(\left|p_{1}\right|>>\left|p_{2}\right|\right)$, e $p<0$ para modos fortemente confinados dentro do guia. Assim, quatro casos distintos podem ser observados para modos lentos: (i) $\varepsilon_{2} \mu_{2}>\varepsilon_{1} \mu_{1}, \mu_{2}>\mu_{1}$. Somente modos ímpares existem abaixo do limiar, $\left(k_{2}^{2}-k_{1}^{2}\right)(d / 2)^{2}<\mu_{1}^{2} / \mu_{2}^{2}$. O valor crítico correspondente da espessura $d$ abaixo do qual modos ímpares existem vale

$$
d_{c r}=\frac{c}{\omega} \frac{\mu_{1}}{\mu_{2} \sqrt{\varepsilon_{2} \mu_{2}-\varepsilon_{1} \mu_{1}}}
$$

O fluxo de energia $P$ e positivo para todos os valores de $d$. Os modos são de ida, i. e., o fluxo de energia total através do guia é codirecionado com o vetor de onda.

(ii) $\varepsilon_{2} \mu_{2}>\varepsilon_{1} \mu_{1}, \mu_{2}<\mu_{1}$. Modos pares existem para todos os valores de $\left(k_{2}^{2}-k_{1}^{2}\right)(d / 2)^{2}$; modos ímpares poderão aparecer somente quando um parâmetro de limiar é excedido, $\left(k_{2}^{2}-k_{1}^{2}\right)(d / 2)^{2}>\mu_{1}{ }^{2} / \mu_{2}{ }^{2}$. O valor crítico da eq. (33) determina o limite mínimo da região de existência de modos ímpares. O fluxo de energia total é negativo para todo $d$, e os modos são contrapropagantes. A energia é, em sua maior parte, localizada dentro do slab.

(iii) $\varepsilon_{2} \mu_{2}<\varepsilon_{1} \mu_{1}, \mu_{2}>\mu_{1}$. Ambos os modos pares e ímpares existem para todo $d$ e $\left(k_{2}^{2}-k_{1}^{2}\right)(d / 2)^{2}$, e os modos são propagantes.

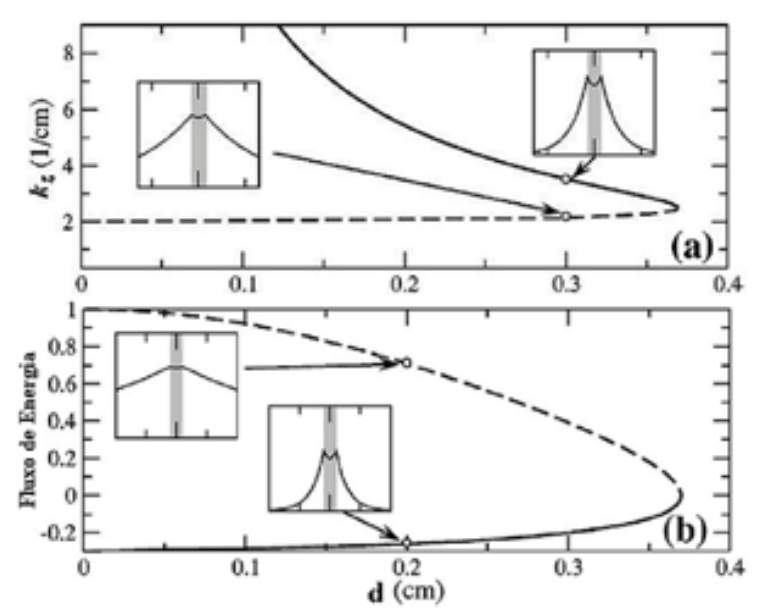

Figura 7. Ondas de superfície em um slab para o caso $\varepsilon_{2} \mu_{2}<\varepsilon_{1} \mu_{1}$ e $\mu_{2}<\mu_{1}$ e, onde se vê em (a) a constante de propagação $k_{z}$ e em (b) o fluxo de energia normalizado $p$ vs o parâmetro de espessura do slab $d$. Linhas sólidas e tracejadas correspondem a modos fortemente e fracamente localizados, respectivamente. Nos quadros, os perfis transversais dos modos. Adaptado da figura 3 de [6]. 
(iv) $\varepsilon_{2} \mu_{2}<\varepsilon_{1} \mu_{1}, \mu_{2}<\mu_{1}$. Somente modos pares existem abaixo de um valor limiar de $\left(k_{2}^{2}-k_{1}^{2}\right)(d / 2)^{2}$ e que pode ser obtido da eq. (18) ou (19). As dependências características do número de onda $k_{z}$, e o fluxo de energia $P$ versus a espessura do slab são mostradas nas figuras 7(a) e 7(b). Para qualquer espessura abaixo de um valor crítico, dois modos sempre coexistem. Um dos modos é propagante e fracamente localizado, enquanto que o outro é contrapropagante e mais confinado. Quando um certo valor de espessura do slab é atingido, os ramos correspondentes a diferentes modos se fundem e o fluxo de energia se anula. Para este caso especial, os fluxos de energia dentro e fora do slab se compensam mutuamente.

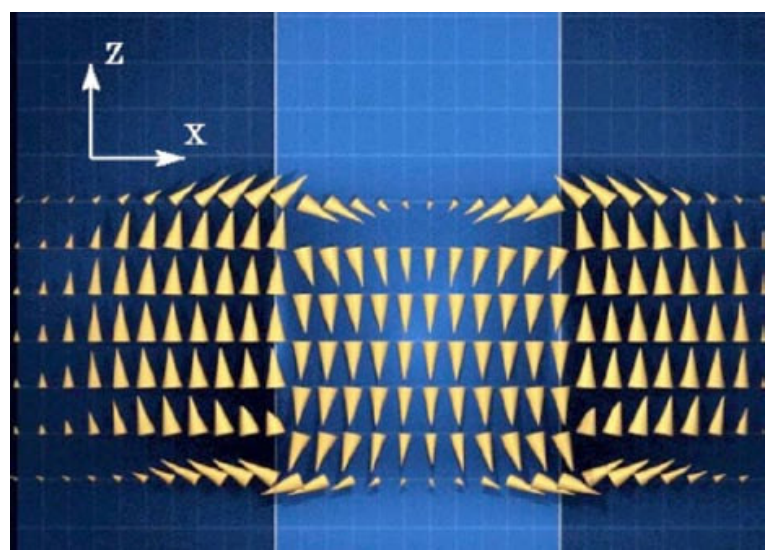

Figura 8. Estrutura do campo do vetor de Poyting em uma onda de superfície localizada propagando-se através de um slab LHM. Adaptado da figura 4 de [6].

Como os fluxos de energia são antiparalelos dentro dos modos guiados, pode parecer, à primeira vista, que tais ondas podem ser mantidas por duas fontes continuamente operando nos terminais opostos do guia-de-onda. Logo, é importante compreender se pacotes de onda de extensões temporais e espaciais finitas podem existir em guias-deonda LHM. Em [6] é apresentada a estrutura característica do fluxo de energia, a partir da média do vetor de Poynting sobre um período da freqüência de um pulso a partir de uma portadora, aqui reproduzido na Fig. 8. Devido à estrutura única de duplo-vórtice do fluxo de energia, a maior parte da energia permanece localizada dentro do pacote de onda, e não se desintegra. A velocidade de grupo é proporciona ao fluxo de energia total $P$, e pode, portanto, ser feito muito baixa ou até mesmo zero, com uma escolha adequada dos parâmetros do guia-de-onda como demonstrado. Por outro lado, a dispersão da velocidade de grupo, o qual determina a taxa de largura de pulso, pode também ser controlada. Esta flexibilidade parece muito promissora para potenciais aplicações [8].

\section{OBSERVAÇÕES FINAIS}

Uma revisão sobre ondas em metamateriais foi feita. Os modos guiados em guias-de-onda preenchidos ou inseridos em um meio metamaterial isotrópico foram determinados. Pode-se mostrar que para números de onda transversais reais, freqüências de corte existem para todos os modos, enquanto que para números de onda transversais imaginários, dependendo dos parâmetros constitutivos, podem existir modos sem cortes. A hierarquia convencional dos modos foi sensivelmente modificada no caso de guias LHM, e pode-se provar que o modo fundamental não existe, o modo de primeira ordem existe somente dentro de uma faixa de valores de $\left(k_{2 x} d / 2\right)$ e desaparece após um valor crítico de $\left(k_{2 x} d / 2\right)$ ser excedido, além de dois modos com o mesmo número de nós poderem coexistir dentro do mesmo guia. Outras propriedades, como a variação de sinal do fluxo de energia, e a existência de diferentes classes de ondas guiadas como uma estrutura interna tipo vórtice, também foram descritas neste trabalho.

\section{AGRADECIMENTOS}

L. A. A. e H. H. F. agradecem ao CNPq (Conselho Nacional de Desenvolvimento Científico e Tecnológico) e FAPEST (Fundação de Amparo à Pesquisa do Estado de São Paulo), H. T. S. agradece ao projeto UTA-Chile $\mathrm{N}^{\circ} 8721-06$.

\section{REFERÊNCIAS}

[1] V.G. Veselago. "The Electrodynamics of substances with simultaneously negative values of $\varepsilon$ and $\mu$ ". Sov. Phys. Usp. Vol. 10, pp. 509-514. 1968.

[2] J.B. Pendry. "Negative refraction makes a perfect lens". Phys. Rev. Lett. Vol 85, pp. 3966-3969. 2000.

[3] H. Torres-Silva. C. Villarroel, P.H. Sakanaka and N. Reggiani. "Electromagnetic properties of a chiral plasma medium". Pramana Journal of Physics. Vol 49, pp. 431-442. 1997.

[4] B.I. Wu, T.M. Grzegorczyk, T. Zhang and J.A. Kong. "Guided modes with imaginary transverse wave number in a slab waveguide with negative permittivity and permeability". J. Appl. Phys. Vol. 93, pp. 9386-9388. 2003. 
[5] A.C. Peacock and G.R. Broderick. "Guided modes in channel waveguides with a negative index of refraction". Optics Express. Vol. 11, pp. 2502-2510. 2003.

[6] I.V. Shadrivov, A.A. Sukhorukov and Y.S. Kivshar. "Guided modes in negative-refractive-index waveguides". Phys. Rev. E. Vol. 67, 057602. 2003.
[7] C. Caloz, C.C. Chang and T. Itoh. "Full-wave verification of the fundamental properties of left-handed materials in waveguide configurations”. J. Appl. Phys. Vol. 90, pp. 54835486. 2001.

[8] D.L. Lee. "Electromagnetic Principles of Integrated Optics". John Wiley \& Sons. New York. 1986. 